\title{
Peer review guidance: a primer for researchers
}

\author{
Olena Zimba1 ${ }^{1 D}$, Armen Yuri Gasparyan² ID \\ ${ }^{1}$ Department of Internal Medicine No. 2, Danylo Halytsky Lviv National Medical University, Lviv, Ukraine \\ ${ }^{2}$ Departments of Rheumatology and Research and Development, Dudley Group NHS Foundation Trust (Teaching Trust of the University \\ of Birmingham, UK), Russells Hall Hospital, Dudley, West Midlands, UK
}

\begin{abstract}
The peer review process is essential for quality checks and validation of journal submissions. Although it has some limitations, including manipulations and biased and unfair evaluations, there is no other alternative to the system. Several peer review models are now practised, with public review being the most appropriate in view of the open science movement. Constructive reviewer comments are increasingly recognised as scholarly contributions which should meet certain ethics and reporting standards. The Publons platform, which is now part of the Web of Science Group (Clarivate Analytics), credits validated reviewer accomplishments and serves as an instrument for selecting and promoting the best reviewers. All authors with relevant profiles may act as reviewers. Adherence to research reporting standards and access to bibliographic databases are recommended to help reviewers draft evidence-based and detailed comments.
\end{abstract}

Key words: research peer review, publishing, periodicals as topic, publication ethics, rheumatology.

\section{Introduction}

The peer review process is essential for evaluating the quality of scholarly works, suggesting corrections, and learning from other authors' mistakes. The principles of peer review are largely based on professionalism, eloquence, and collegiate attitude. As such, reviewing journal submissions is a privilege and responsibility for 'elite' research fellows who contribute to their professional societies and add value by voluntarily sharing their knowledge and experience.

Since the launch of the first academic periodicals back in 1665, the peer review has been mandatory for validating scientific facts, selecting influential works, and minimizing chances of publishing erroneous research reports [1]. Over the past centuries, peer review models have evolved from single-handed editorial evaluations to collegial discussions, with numerous strengths and inevitable limitations of each practised model [2, 3]. With multiplication of periodicals and editorial management platforms, the reviewer pool has expanded and internationalized. Various sets of rules have been proposed to select skilled reviewers and employ globally acceptable tools and language styles [4, 5].

In the era of digitization, the ethical dimension of the peer review has emerged, necessitating involvement of peers with full understanding of research and publication ethics to exclude unethical articles from the pool of evidence-based research and reviews [6]. In the time of the COVID-19 pandemic, some, if not most, journals face the unavailability of skilled reviewers, resulting in an unprecedented increase of articles without a history of peer review or those with surprisingly short evaluation timelines [7].

\section{Editorial recommendations and the best reviewers}

Guidance on peer review and selection of reviewers is currently available in the recommendations of global editorial associations which can be consulted by journal editors for updating their ethics statements and by research managers for crediting the evaluators. The International Committee on Medical Journal Editors (ICMJE) qualifies peer review as a continuation of the scientific 
Table I. Structure of a reviewer comment to be forwarded to authors

\begin{tabular}{|l|c|}
\hline Section & Notes \\
\hline Introductory line & Summarizes the overall impression about the manuscript validity and implications \\
\hline $\begin{array}{l}\text { Evaluation of the title, } \\
\text { abstract and keywords }\end{array}$ & $\begin{array}{r}\text { Evaluates the title correctness and completeness, inclusion of all relevant keywords, } \\
\text { study design terms, information load, and relevance of the abstract }\end{array}$ \\
\hline Major comments & $\begin{array}{r}\text { Specifically analyses each manuscript part in line with available research reporting standards, } \\
\text { supports all suggestions with solid evidence, weighs novelty of hypotheses and methodological } \\
\text { rigour, highlights the choice of study design, points to missing/incomplete ethics approval } \\
\text { statements, rights to re-use graphics, accuracy and completeness of statistical analyses, } \\
\text { professionalism of bibliographic searches and inclusion of updated and relevant references }\end{array}$ \\
\hline Minor comments & $\begin{array}{r}\text { Identifies language mistakes, typos, inappropriate format of graphics and references, } \\
\text { length of texts and tables, use of supplementary material, unusual sections and order, complete- } \\
\text { ness of scholarly contribution, conflict of interest, and funding statements }\end{array}$ \\
\hline Concluding remarks & Reflects on take-home messages and implications \\
\hline
\end{tabular}

process that should involve experts who are able to timely respond to reviewer invitations, submitting unbiased and constructive comments, and keeping confidentiality [8].

The reviewer roles and responsibilities are listed in the updated recommendations of the Council of Science Editors (CSE) [9] where ethical conduct is viewed as a premise of the quality evaluations. The Committee on Publication Ethics (COPE) further emphasizes editorial strategies that ensure transparent and unbiased reviewer evaluations by trained professionals [10]. Finally, the World Association of Medical Editors (WAME) prioritizes selecting the best reviewers with validated profiles to avoid substandard or fraudulent reviewer comments [11]. Accordingly, the Sarajevo Declaration on Integrity and Visibility of Scholarly Publications encourages reviewers to register with the Open Researcher and Contributor ID (ORCID) platform to validate and publicize their scholarly activities [12].

Although the best reviewer criteria are not listed in the editorial recommendations, it is apparent that the manuscript evaluators should be active researchers with extensive experience in the subject matter and an impressive list of relevant and recent publications [13]. All authors embarking on an academic career and publishing articles with active contact details can be involved in the evaluation of others' scholarly works [14] Ideally, the reviewers should be peers of the manuscript authors with equal scholarly ranks and credentials.

However, journal editors may employ schemes that engage junior research fellows as co-reviewers along with their mentors and senior fellows [15]. Such a scheme is successfully practised within the framework of the Emerging EULAR (European League Against Rheumatism) Network (EMEUNET) where seasoned authors (mentors) train ongoing researchers (mentees) how to evaluate submissions to the top rheumatology journals and select the best evaluators for regular contributors to these journals [16].
The awareness of the EQUATOR Network reporting standards may help the reviewers to evaluate methodology and suggest related revisions. Statistical skills help the reviewers to detect basic mistakes and suggest additional analyses. For example, scanning data presentation and revealing mistakes in the presentation of means and standard deviations often prompt re-analyses of distributions and replacement of parametric tests with non-parametric ones $[17,18]$.

\section{Constructive reviewer comments}

The main goal of the peer review is to support authors in their attempt to publish ethically sound and professionally validated works that may attract readers' attention and positively influence healthcare research and practice. As such, an optimal reviewer comment has to comprehensively examine all parts of the research and review work (Table I). The best reviewers are viewed as contributors who guide authors on how to correct mistakes, discuss study limitations, and highlight its strengths [19].

Some of the currently practised review models are well positioned to help authors reveal and correct their mistakes at pre- or post-publication stages (Table II). The global move toward open science is particularly instrumental for increasing the quality and transparency of reviewer contributions.

Since there are no universally acceptable criteria for selecting reviewers and structuring their comments, instructions of all peer-reviewed journal should specify priorities, models, and expected review outcomes [20]. Monitoring and reporting average peer review timelines is also required to encourage timely evaluations and avoid delays. Depending on journal policies and article types, the first round of peer review may last from a few days to a few weeks. The fast-track review (up to 3 days) is practised by some top journals which process clinical trial reports and other priority items. 
Table II. Advantages and disadvantages of common manuscript evaluation models

\begin{tabular}{|c|c|c|}
\hline Models & Advantages & Disadvantages \\
\hline $\begin{array}{l}\text { In-house (internal) } \\
\text { editorial review }\end{array}$ & $\begin{array}{c}\text { Allows detection of major flaws and errors that } \\
\text { justify outright rejections; rarely, outstanding } \\
\text { manuscripts are accepted without delays }\end{array}$ & $\begin{array}{l}\text { Journal staff evaluations may be biased; manu- } \\
\text { script acceptance without external review may } \\
\text { raise concerns of soft quality checks }\end{array}$ \\
\hline $\begin{array}{l}\text { Single-blind peer } \\
\text { review }\end{array}$ & $\begin{array}{l}\text { Masking reviewer identity prevents personal con- } \\
\text { flicts in small (closed) professional communities }\end{array}$ & $\begin{array}{c}\text { Reviewer access to author profiles may result in } \\
\text { biased and subjective evaluations }\end{array}$ \\
\hline $\begin{array}{l}\text { Double-blind peer } \\
\text { review }\end{array}$ & $\begin{array}{c}\text { Concealing author and reviewer identities } \\
\text { prevents biased evaluations, particularly in small } \\
\text { communities }\end{array}$ & $\begin{array}{l}\text { Masking all identifying information is technically } \\
\text { burdensome and not always possible }\end{array}$ \\
\hline $\begin{array}{l}\text { Open (public) peer } \\
\text { review }\end{array}$ & $\begin{array}{l}\text { May increase quality, objectivity, and accountabil- } \\
\text { ity of reviewer evaluations; it is now part of open } \\
\text { science culture }\end{array}$ & $\begin{array}{l}\text { Peers who do not wish to disclose their identity } \\
\text { may decline reviewer invitations }\end{array}$ \\
\hline $\begin{array}{l}\text { Post-publication } \\
\text { open peer review }\end{array}$ & $\begin{array}{l}\text { May accelerate dissemination of influential } \\
\text { reports in line with the concept "publish first, } \\
\text { judge later"; this concept is practised by some } \\
\text { open-access journals (e.g., F1000 Research) }\end{array}$ & $\begin{array}{l}\text { Not all manuscripts benefit from open dissemina- } \\
\text { tion without peers' input; post-publication review } \\
\text { may delay detection of minor or major mistakes }\end{array}$ \\
\hline $\begin{array}{l}\text { Post-publication social } \\
\text { media commenting }\end{array}$ & $\begin{array}{l}\text { May reveal some mistakes and misconduct and } \\
\text { improve public perception of article implications }\end{array}$ & $\begin{array}{l}\text { Not all communities use social media for com- } \\
\text { menting and other academic purposes }\end{array}$ \\
\hline
\end{tabular}

In exceptional cases, reviewer contributions may result in substantive changes, appreciated by authors in the official acknowledgments. In most cases, however, reviewers should avoid engaging in the authors' research and writing. They should refrain from instructing the authors on additional tests and data collection as these may delay publication of original submissions with conclusive results.

Established publishers often employ advanced editorial management systems that support reviewers by providing instantaneous access to the review instructions, online structured forms, and some bibliographic databases. Such support enables drafting of evidence-based comments that examine the novelty, ethical soundness, and implications of the reviewed manuscripts [21].

Encouraging reviewers to submit their recommendations on manuscript acceptance/rejection and related editorial tasks is now a common practice. Skilled reviewers may prompt the editors to reject or transfer manuscripts which fall outside the journal scope, perform additional ethics checks, and minimize chances of publishing erroneous and unethical articles. They may also raise concerns over the editorial strategies in their comments to the editors.

Since reviewer and editor roles are distinct, reviewer recommendations are aimed at helping editors, but not at replacing their decision-making functions. The final decisions rest with handling editors. Handling editors weigh not only reviewer comments, but also priorities related to article types and geographic origins, space limitations in certain periods, and envisaged influence in terms of social media attention and citations. This is why rejections of even flawless manuscripts are likely at early rounds of internal and external evaluations across most peer-reviewed journals.

Reviewers are often requested to comment on language correctness and overall readability of the evaluated manuscripts. Given the wide availability of in-house and external editing services, reviewer comments on language mistakes and typos are categorized as minor. At the same time, non-Anglophone experts' poor language skills often exclude them from contributing to the peer review in most influential journals [22]. Comments should be properly edited to convey messages in positive or neutral tones, express ideas of varying degrees of certainty, and present logical order of words, sentences, and paragraphs [23, 24]. Consulting linguists on communication culture, passing advanced language courses, and honing commenting skills may increase the overall quality and appeal of the reviewer accomplishments [5, 25].

\section{Peer reviewer credits}

Various crediting mechanisms have been proposed to motivate reviewers and maintain the integrity of science communication [26]. Annual reviewer acknowledgments are widely practised for naming manuscript evaluators and appreciating their scholarly contributions. Given the need to weigh reviewer contributions, some journal editors distinguish 'elite' reviewers with numerous evaluations and award those with timely and outstanding accomplishments [27]. Such targeted recognition ensures ethical soundness of the peer review and facilitates promotion of the best candidates for grant funding and academic job appointments [28]. 
Also, large publishers and learned societies issue certificates of excellence in reviewing which may include Continuing Professional Development (CPD) points [29]. Finally, an entirely new crediting mechanism is proposed to award bonus points to active reviewers who may collect, transfer, and use these points to discount gold open-access charges within the publisher consortia [30].

With the launch of Publons (http://publons.com/) and its integration with Web of Science Group (Clarivate Analytics), reviewer recognition has become a matter of scientific prestige. Reviewers can now freely open their Publons accounts and record their contributions to online journals with Digital Object Identifiers (DOI). Journal editors, in turn, may generate official reviewer acknowledgments and encourage reviewers to forward them to Publons for building up individual reviewer and journal profiles. All published articles maintain e-links to their review records and post-publication promotion on social media, allowing the reviewers to continuously track expert evaluations and comments. A paid-up partnership is also available to journals and publishers for automatically transferring peer-review records to Publons upon mutually acceptable arrangements.

Listing reviewer accomplishments on an individual Publons profile showcases scholarly contributions of the account holder. The reviewer accomplishments placed next to the account holders' own articles and editorial accomplishments point to the diversity of scholarly contributions. Researchers may establish links between their Publons and ORCID accounts to further benefit from complementary services of both platforms. Publons Academy (https://publons.com/community/academy/) additionally offers an online training course to novice researchers who may improve their reviewing skills under the guidance of experienced mentors and journal editors. Finally, journal editors may conduct searches through the Publons platform to select the best reviewers across academic disciplines.

\section{Peer review ethics}

Prior to accepting reviewer invitations, scholars need to weigh a number of factors which may compromise their evaluations. First of all, they are required to accept the reviewer invitations if they are capable of timely submitting their comments. Peer review timelines depend on article type and vary widely across journals. The rules of transparent publishing necessitate recording manuscript submission and acceptance dates in article footnotes to inform readers of the evaluation speed and to help investigators in the event of multiple unethical submissions. Timely reviewer accomplishments often enable fast publication of valuable works with positive implications for healthcare. Unjustifiably long peer re- view, on the contrary, delays dissemination of influential reports and results in ethical misconduct, such as plagiarism of a manuscript under evaluation [31].

In the times of proliferation of open-access journals relying on article processing charges, unjustifiably short review may point to the absence of quality evaluation and apparently 'predatory' publishing practice [32, 33]. Authors when choosing their target journals should take into account the peer review strategy and associated timelines to avoid substandard periodicals.

Reviewer primary interests (unbiased evaluation of manuscripts) may come into conflict with secondary interests (promotion of their own scholarly works), necessitating disclosures by filling in related parts in the online reviewer window or uploading the ICMJE conflict of interest forms. Biomedical reviewers, who are directly or indirectly supported by the pharmaceutical industry, may encounter conflicts while evaluating drug research. Such instances require explicit disclosures of conflicts and/or rejections of reviewer invitations.

Journal editors are obliged to employ mechanisms for disclosing reviewer financial and non-financial conflicts of interest to avoid processing of biased comments [34]. They should also cautiously process negative comments that oppose dissenting, but still valid, scientific ideas [35]. Reviewer conflicts that stem from academic activities in a competitive environment may introduce biases, resulting in unfair rejections of manuscripts with opposing concepts, results, and interpretations. The same academic conflicts may lead to coercive reviewer selfcitations, forcing authors to incorporate suggested reviewer references or face negative feedback and an unjustified rejection [36]. Notably, several publisher investigations have demonstrated a global scale of such misconduct, involving some highly cited researchers and top scientific journals [37].

Fake peer review, an extreme example of conflict of interest, is another form of misconduct that has surfaced in the time of mass proliferation of gold openaccess journals and publication of articles without quality checks [38]. Fake reviews are generated by manipulating authors and commercial editing agencies with full access to their own manuscripts and peer review evaluations in the journal editorial management systems. The sole aim of these reviews is to break the manuscript evaluation process and to pave the way for publication of pseudoscientific articles. Authors of these articles are often supported by funds intended for the growth of science in non-Anglophone countries [39]. Iranian and Chinese authors are often caught submitting fake reviews, resulting in mass retractions by large publishers [38]. Several suggestions have been made to overcome this issue, with assigning independent reviewers and 
requesting their ORCID IDs viewed as the most practical options [40].

\section{Conclusions}

The peer review process is regulated by publishers and editors, enforcing updated global editorial recommendations. Selecting the best reviewers and providing authors with constructive comments may improve the quality of published articles. Reviewers are selected in view of their professional backgrounds and skills in research reporting, statistics, ethics, and language. Quality reviewer comments attract superior submissions and add to the journal's scientific prestige [41].

In the era of digitization and open science, various online tools and platforms are available to upgrade the peer review and credit experts for their scholarly contributions. With its links to the ORCID platform and socia media channels, Publons now offers the optimal model for crediting and keeping track of the best and most active reviewers. Publons Academy additionally offers online training for novice researchers who may benefit from the experience of their mentoring editors. Overall, reviewer training in how to evaluate journal submissions and avoid related misconduct is an important process, which some indexed journals are experimenting with [42].

The timelines and rigour of the peer review may change during the current pandemic. However, journal editors should mobilize their resources to avoid publication of unchecked and misleading reports. Additional efforts are required to monitor published contents and encourage readers to post their comments on publishers' online platforms (blogs) and other social media channels $[43,44]$.

The authors declare no conflict of interest.

\section{References}

1. Banks D. Thoughts on publishing the research article over the centuries. Publications 2018; 6: 10, DOI: 10.3390/publications6010010.

2. Jana S. A history and development of peer-review process. Ann Libr Inf Stud 2019; 66: 152-162.

3. Peer review should be an honest, but collegial, conversation Nature 2020; 582: 314, DOI: 10.1038/d41586-020-01622-z.

4. Wicherts JM. Peer Review Quality and Transparency of the Peer-Review Process in Open Access and Subscription Journals. PLoS One 2016; 11: e0147913, DOI: 10.1371/journal. pone. 0147913 .

5. Whang Y. Reviewing a journal article with clarity and politeness: Key language tips for non-native English-speaking reviewers. Sci Ed 2020; 7: 204-208, DOI: 10.6087/kcse.220.

6. Lazarides MK, Georgiadis GS, Papanas N. Do's and Don'ts for a Good Reviewer of Scientific Papers: A Beginner's Brief
Decalogue. Int J Low Extrem Wounds 2020; 19: 227-229, DOI: 10.1177/1534734620924349.

7. Kambakamba P, Geoghegan J, Hoti E. The peer review at high risk from COVID-19 - are we socially distancing from scientific quality control? Br J Surg 2020; 107: e334-e335, DOI: 10.1002/ bjs.11785.

8. Recommendations for the Conduct, Reporting, Editing, and Publication of Scholarly Work in Medical Journals Updated December 2019. Available from: http://www.icmje.org/icmje-recommendations.pdf [Accessed: 5.12.2020].

9. CSE's White Paper on Promoting Integrity in Scientific Journal Publications. Available from: https://www.councilscienceeditors.org/wp-content/uploads/CSE-White-Paper_2018-update-050618.pdf [Accessed: 5.12.2020].

10. Core practices. Available from: https://publicationethics.org/ core-practices [Accessed: 10.12.2020].

11. Best Practices for Peer Reviewer Selection and Contact to Prevent Peer Review Manipulation by Authors. Available from: http://wame.org/best-practices-for-peer-reviewer-selection-and-contact-to-prevent-peer-review-manipulation-byauthors [Accessed: 5.12.2020].

12. Mašić I, Begić E, Donev DM, et al. Sarajevo Declaration on Integrity and Visibility of Scholarly Publications. Croat Med J 2016; 57: 527-529, DOI: 10.3325/cmj.2016.57.527.

13. Gasparyan AY, Kitas GD. Best peer reviewers and the quality of peer review in biomedical journals. Croat Med J 2012; 53: 386-389, DOI: 10.3325/cmj.2012.53.386.

14. Gasparyan AY, Yessirkepov M, Gorin SV, Kitas GD. Educating science editors: is there a comprehensive strategy? Croat Med J 2014; 55: 672-675, DOI: 10.3325/cmj.2014.55.672.

15. Recognizing the involvement of early-career researchers in peer review. Nat Rev Endocrinol 2020; 16: 535.

16. Rodríguez-Carrio J, Putrik P, Sepriano A, et al. Improving the peer review skills of young rheumatologists and researchers in rheumatology: the EMEUNET Peer Review Mentoring Program. RMD Open 2018; 4: e000619 DOI: 10.1136/rmdopen2017-000619.

17. Habibzadeh F. Statistical Data Editing in Scientific Articles. J Korean Med Sci 2017; 32: 1072-1076, DOI: 10.3346/jkms. 2017.32.7.1072.

18. Misra DP, Agarwal V. Integrity of clinical research conduct, reporting, publishing, and post-publication promotion in rheumatology. Clin Rheumatol 2020; 39: 1049-1060, DOI: 10.1007/ s10067-020-04965-0.

19. Araújo CG. Peer review: a constantly-evolving scientific process. Arq Bras Cardiol 2012; 98: e32-35, DOI: 10.1590/S0066782X2012000200017.

20. Gasparyan AY, Ayvazyan L, Gorin SV, Kitas GD. Upgrading instructions for authors of scholarly journals. Croat Med J 2014; 55: 271-280, DOI: $10.3325 / \mathrm{cmj} .2014 .55 .271$.

21. Nahlen D, Clark S. The Publisher's Perspective on Journal and Book Publishing. Semin Oncol Nurs 2018; 34: 381-385, DOI: 10.1016/j.soncn.2018.09.006.

22. Masukume G, Grech V. The Lancet peer reviewers: global pattern and distribution. Lancet 2018; 391: 2603-2604, DOI: 10.1016/S0140-6736(18)31136-X.

23. Mavrogenis AF, Quaile A, Scarlat MM. The good, the bad and the rude peer-review. Int Orthop 2020; 44: 413-415. 
24. If you can't be kind in peer review, be neutral. Nature 2020, DOI: 10.1038/d41586-020-03394-y [Online ahead of print].

25. Yakhontova T. Conventions of English Research Discourse and the Writing of Non-Anglophone Authors. J Korean Med Sci 2020; 35: e331, DOI: 10.3346/jkms.2020.35.e331.

26. Gasparyan AY, Gerasimov AN, Voronov AA, Kitas GD. Rewarding peer reviewers: maintaining the integrity of science communication. J Korean Med Sci 2015; 30: 360-364 DOI: 10.3346/ jkms.2015.30.4.360.

27. Misra DP, Ravindran V. Peer review in academic publishing: threats and challenges. J R Coll Physicians Edinb 2019; 49 99-100, DOI: 10.4997/JRCPE.2019.201.

28. Klein JS. Editor's Recognition Awards. Radiographics 2020; 40: 305, DOI: 10.1148/rg.2020204001.

29. Riley BJ, Jones R. Peer review: acknowledging its value and recognising the reviewers. Br J Gen Pract 2016; 66: 629-630, DOI: 10.3399/bjgp16X688285.

30. Gurwitz D. Peer review: Award bonus points to motivate reviewers. Nature 2017; 542: 414, DOI: 10.1038/542414d.

31. Mehregan M. Ethical Reviewers are Essential for Scholarly Journals for Timely Processing of Submissions and Avoiding Retractions. J Korean Med Sci 2019; 34: e41, DOI: 10.3346/ jkms.2019.34.e41.

32. Fernandez-Llimos F. Open access, predatory publishing and peer-review. Pharm Pract (Granada) 2014; 12: 427, DOI: 10.4321/ s1886-36552014000100001.

33. McCann TV, Polacsek M. False gold: Safely navigating open access publishing to avoid predatory publishers and journals. J Adv Nurs 2018; 74: 809-817, DOI: 10.1111/jan.13483.

34. Gasparyan AY, Ayvazyan L, Akazhanov NA, Kitas GD. Conflicts of interest in biomedical publications: considerations for authors, peer reviewers, and editors. Croat Med J 2013; 54 600-608, DOI: 10.3325/cmj.2013.54.600.
35. Hirsch JA, Manchikanti L, Albuquerque FC, et al. The peer review process: a primer for JNIS readers. J Neurointerv Surg 2017; 9: e3-e6, DOI: 10.1136/neurintsurg-2015-011781.

36. Thombs BD, Levis AW, Razykov I, et al. Potentially coercive self-citation by peer reviewers: a cross-sectional study. J Psychosom Res 2015; 78: 1-6, DOI: 10.1016/j.jpsychores. 2014.09.015.

37. Van Noorden R. Highly cited researcher banned from journal board for citation abuse. Nature 2020; 578: 200-201, DOI: 10.1038/d41586-020-00335-7.

38. Rivera H. Fake Peer Review and Inappropriate Authorship Are Real Evils. J Korean Med Sci 2018; 34: e6, DOI: 10.3346/ jkms.2019.34.e6.

39. Cyranoski D. China cracks down on fake peer reviews. Nature 2017; 546: 464, DOI: 10.1038/546464a.

40. Ferguson C, Marcus A, Oransky I. Publishing: The peer-review scam. Nature 2014; 515: 480-482, DOI: 10.1038/515480a.

41. Gasparyan AY. Choosing the target journal: do authors need a comprehensive approach? J Korean Med Sci 2013; 28 : 1117-1179, DOI: 10.3346/jkms.2013.28.8.1117.

42. Gregory AT, Denniss AR. Everything You Need to Know About Peer Review - The Good, The Bad and The Ugly. Heart Lung Circ 2019; 28: 1148-1153, DOI: 10.1016/j.hlc.2019.05.171

43. Bauchner H, Fontanarosa PB, Golub RM. Editorial Evaluation and Peer Review During a Pandemic: How Journals Maintain Standards. JAMA 2020; 324: 453-454, DOI: 10.1001/jama. 2020.11764

44. Goel A, Gupta L. Social Media in the Times of COVID-19. J Clin Rheumatol 2020; 26: 220-223, DOI: 10.1097/rhu.00000 00000001508. 